\title{
The experimental study on the migration of human mesenchymal stem cells and prostate cancer
}

\author{
Gongxian Wang ${ }^{1}$, Yang Wang ${ }^{1}$, HongLin $\mathrm{Hu}^{1}$, Yian Zhan ${ }^{1}$ \\ ${ }^{l}$ Department of Urology, The First Affiliated Hospital of Nanchang University, Nanchang, China
}

Our purposeis to investigate the migration behavior of human mesenchymal stem cells (hMSCs) in response to prostate cancer. The human prostate cancer cell line PC-3 (PC-3) were injected into the flank or axillary of Severe combined immunodeficiency (SCID) mice subcutaneously to establish human prostate cancer xenograft models. hMSCs were harvested from donor's ribs of human cadaver renal transplantation and separated by density gradient centrifuge. hMSCs between passages 4 to 6 were labeled with 4,6-Diamidino-2-phenylindole (DAPI). DAPI-labeled hMSCs were injected into the bearing cancer SCID mice by tail vein (five doses $\left(10^{6}\right.$ cells/dose) of labeled MSCs over a 15 20-day period) or $0.5 \mathrm{~cm}$ away from peritumor (one dose of $10^{6}$ cells) subcutaneously. 17 days after injection by tail vein, 7 days, 10 days and 14 days after injection by peritumor subcutaneously, the mice were killed and their tumors, livers, lungs, spleens and kidneys were harvested. Frozen sections and paraffin sections were used to observe the distribution of exogenous DAPI-labeled hMSCs in vivo by fluorescence microscope. We measured the expression of prostate specific antigen (PSA) and prostate-specific membrane antigen (PSMA) in tumor tissue by immunohistochemistry. We developed a two-dimensional in vitro model with $1 \%$ low-melt agarose dissolved in Dulbecco modified Eagle medium/F12. The PC-3 cells and hMSCs were plated in the exposed area of the model. Then to observe the adhesion between PC-3 cells and hMSCs. In SCID mice injected with PC-3 subcutaneously,the tumor take rate was $83.3 \%$. The tumors were identified by pathology. The tumors were negative for PSA and PSMA by immunohistochemistry. 17 days after injection hMSCs by tail vein, 7 days, 10 days and 14 days after injection hMSCs by peritumor subcutaneously, DAPI-labeled hMSCs with blue nuclei were distributed extensively in the tumors. But no blue nucleus was seen in the livers, lungs, spleens and kidneys. The tumor growth was accelerated after injection hMSCs. In vitro model, the PC-3 cells adhered to hMSCs after a day coculture. A significant increase in the adhesion of PC-3 cells to hMSCs was found at $4 \sim 7$ days cocultures. Exogenous purified hMSCs can migrate to prostate cancer microenviroment in vivo. The $\mathrm{PC}-3$ cells can adhere to hMSCs in vitro.

Keywords: human mesenchymal stem cells, PC-3, prostate cancer microenviroment, migration

Cell Research (2008) 18:s67. doi: 10.1038/cr.2008.157; published online 4 August 2008

Correspondence: Gong-xian Wang

E-mail: wanggx-mr@126.com 